\title{
Conocimiento del entorno natural y política ambiental para la conservación de bromelias epífitas en Santa Catarina Ixtepeji, Oaxaca, México
}

\author{
Knowledge of the natural environment and environmental policy for the conservation \\ of epiphytic bromeliads in Santa Catarina Ixtepeji, Oaxaca, Mexico
}

\begin{abstract}
Elia Méndez-García ${ }^{1}$, Demetria Mondragón-Chaparro ${ }^{\mathbf{1}}$, Laura Gómez-Hernández ${ }^{\mathbf{1}}$
${ }^{1}$ Profesoras-investigadoras del Instituto Politécnico Nacional, adscritas al Centro Interdisciplinario de Investigación para el Desarrollo Integral Regional Unidad Oaxaca, México. Hornos Núm. 1003 Col. Noche Buena, Xoxocotlán, Oaxaca. C. P. 71230 Contacto: mendezeli@hotmail.com
\end{abstract}

\section{Resumen}

La relación cercana con el entorno natural permite a diferentes grupos humanos desarrollar conocimiento sobre él, su aprovechamiento para la reproducción de la vida, su cuidado y defensa. En este trabajo, se analiza la importancia del contacto con los recursos forestales para su conservación, particularmente sobre las bromelias epífitas, y la influencia de la política ambiental, que en aras de fomentar la conservación, pueden incidir en la separación de las poblaciones de su medio ambiente y favorecer el desconocimiento sobre el bosque en las generaciones juveniles e infantiles. Se discuten los resultados de un estudio etnobotánico con niños y adolescentes de Santa Catarina Ixtepeji, Oaxaca, México, en 2015. Se resalta la expresión constante de los participantes sobre la prohibición y sanción de las autoridades comunales e instituciones federales del uso de sus recursos naturales como medida de 


\section{http://revistainvestigacionacademicasinfrontera.com}

conservación. Se sugiere la generación de herramientas bioculturales que fortalezcan la relación con los recursos naturales de la comunidad en los jóvenes y niños.

Palabras clave: política ambiental, etnobotánica, bromelias epífitas.

\section{Summary}

The close relationship with the natural environment allows different human groups to develop knowledge about it, its use for the reproduction of life, its care and defense. In this paper, the importance of contact with forest resources for their conservation, particularly on epiphytic bromeliads, and the influence of environmental policy, which in order to promote conservation, are analyzed. Environment and promote ignorance about the forest in the youth and children's generations. The results of an ethnobotanical study with children and adolescents from Santa Catarina Ixtepeji, Oaxaca, Mexico, in 2015 are discussed. The participants' constant expression of the prohibition and sanction of communal authorities and federal institutions regarding the use of their natural resources As a conservation measure. It is suggested the generation of biocultural tools that strengthen the relationship with the natural resources of the community in the youth and children.

Keywords: environmental policy, ethnobotany, epiphytic bromeliads

\section{Introducción}

La importancia ecológica de las bromelias epífitas ha sido ampliamente descrita, ya que intervienen en los flujos hídricos y nutrimentales, incrementan la biodiversidad, (aproximadamente 1700 especies epífitas), y proveen gran cantidad de microambientes a insectos, anfibios, microorganismos, entre otros. No obstante, también tienen una gran relevancia cultural, pues han sido ampliamente usadas históricamente en rituales religiosos, como plantas de ornato o con fines medicinales (Rees 1976, Rauh 1992, Wolf \& Konings 2001).

En el estado de Oaxaca, uno de los usos más frecuentes es su utilización para el adorno de los nacimientos. Durante el mes de diciembre, existe un mercado establecido para la venta de bromelias epífitas, que ejerce una presión de recolección sobre las poblaciones naturales 


\section{http://revistainvestigacionacademicasinfrontera.com}

ISSN: 2007-8870

en los alrededores de los Valles Centrales de Oaxaca. Ixtepejí, Las Guacamayas y Nochixtlán son principalmente los lugares de mayor extracción con este fin comercial y cultural en esta temporada (Mondragón, 2008).

De acuerdo con estudios poblacionales de estas plantas, la extracción de bromelias epífitas de los bosques cercanos a la ciudad de Oaxaca amenaza su permanencia en las poblaciones naturales (Mondragón, 2008). De ahí que sean necesarias las medidas para su conservación y asegurar su permanencia en el ecosistema. Sin embargo, esta actividad se realiza desde hace más de 30 años, contribuye al mantenimiento de una de las tradiciones más antiguas de México (la puesta de nacimientos) y representa una fuente de ingreso extra en una temporada crítica en la economía familiar (Rees 1976, Mondragón, 2008). Por lo tanto, el plan de manejo debe considerar estos aspectos socioeconómicos y culturales, puesto que su simple prohibición afectaría la economía de las familias de colectores y pondría en riesgo la pérdida de tradiciones.

En estudios etnobotánicos previos sobre las bromelias epífitas en Santa Catarina Ixtepeji, (Mondragón \& Villa, 2008), se describieron las características socioculturales de los colectores a quienes deben enfocarse los planes de manejo y conservación: mujeres mayores de 30 años con educación elemental. Sin embrago, no abordó la percepción de las generaciones infantiles y juveniles sobre la conservación de las bromelias. En este sentido, era necesario saber cuál es el conocimiento y compromiso que se va desarrollando en los niños y adolescentes de esta comunidad, ya que estas generaciones serán las que tomarán las decisiones para la conservación de este recurso.

En este trabajo, se discuten los resultados más importantes del estudio etnobotánico referentes a las nociones de conservación de las bromelias espífitas del bosque en las generaciones juveniles e infantiles en Ixtepeji, Oaxaca, México, en relación con los posibles efectos negativos de las políticas públicas ambientales. Para ello, en primer lugar, presentamos una perspectiva crítica del propósito y dificultades de la aplicación de la política pública ambiental en México en los debates recientes bajo el enfoque de la etnobiodiversidad. En segundo lugar, se describe la metodología: el lugar de estudio como ejemplo de la selvicultura comunitaria y pensándolo dentro de las disyuntivas entre la conservación y las 


\section{http://revistainvestigacionacademicasinfrontera.com}

ISSN: 2007-8870

tradiciones culturales, así como las características del estudio etnobotánico. En tercer lugar, los resultados y discusión, profundizando en los peligros de la percepción de la conservación como prohibición, y por ende, el alejamiento del bosque. Finalmente, a manera de conclusión, se propone la generación de materiales para acercar a los niños y jóvenes al conocimiento de la fenología de las bromelias epífitas; materiales que pueden servir como una herramienta biocultural.

\section{Legislación ambiental mexicana}

La legislación en materia ambiental en México está dirigida hacia una política basada en el "desarrollo sustentable", definido en el marco de la Conferencia de las Naciones Unidas sobre el Medio Ambiente y el Desarrollo, celebrada en Río de Janeiro en 1992, como una noción que involucra tres dimensiones: económica, social y ambiental. Es decir, se busca garantizar no sólo la satisfacción de las necesidades y el bienestar de las generaciones presentes, sino también el de las futuras. En el contexto interno de los Estados, el desarrollo sustentable debe asumirse a través de políticas públicas orientadas a lograr el equilibrio entre desarrollo, tutela ambiental y abatimiento de la pobreza, ya que estos elementos son fundamentales para asegurar un desarrollo humano con una mejor calidad de vida, entendida como crecimiento espiritual y humano, y no sólo en función del aspecto cuantitativo y material, donde la economía es el factor prioritario (Anglés- Hernández, 2008).

Los esfuerzos de la legislación ambiental mexicana encaminada a la construcción de la sustentabilidad están expresos en la elaboración e instrumentación de varias leyes: la Ley General del Equilibrio Ecológico y Protección al Ambiente (LGEEPA) de 1988, la Ley General de Desarrollo Forestal Sustentable (LGDFS) de 2003 y su reglamento publicado en 2005. Las bromelias epífitas se consideran un Recurso Forestal No Maderable (RFNM), por lo que existen otras normatividades con este mismo enfoque de conservación y sustentabilidad, como la Ley General de Vida Silvestre (LGVS), en 2000 y su reglamento publicado en 2016.

Las Unidades de Manejo de Vida Silvestre (UMA) están considerados como espacios para promover esquemas alternativos de producción compatibles con la protección del 


\section{http://revistainvestigacionacademicasinfrontera.com}

ISSN: 2007-8870

ambiente, a través del uso racional, ordenado y planificado de los recursos naturales renovables en ellas contenidos, y que frenan o revierten los procesos de deterioro ambiental, y que junto con los Ordenamientos Ecológicos del Territorio (OET) y la creación de Áreas Naturales Protegidas (ANP), constituyen uno de los principales instrumentos para la conservación de la biodiversidad del país (Gallina, 2008). Es importante hacer la precisión de que una UMA se otorga solamente para especies incluidas en la NOM-059-SEMARNAT2010, que determina las especies en riesgo de extinción. También existen otros permisos de aprovechamiento para especies no incluidas en ella, reguladas por la SEMARNAT.

\section{Perspectiva crítica a las políticas ambientales}

La política ambiental mexicana vio en las UMAs la síntesis para superar el largo debate internacional entre aprovechadores y conservacionistas, del cual no se pretende profundizar aquí. Sólo se apuntan las críticas más fuertes a la perspectiva conservacionista que, por un lado, abren el camino para las propuestas etno dentro del debate mexicano en sus dos grandes líneas: etnobiología con Efraín Hernández-X y etnoecolgía con Víctor Manuel Toledo, cuya mirada común está en mostrar la necesidad de recuperar la influencia determinante de las comunidades humanas, principalmente indígenas, que habitan los territorios donde realizan estudios biológicos y ecológicos. En general, pensar el ámbito biocultural, que implica una postura integral de entender la naturaleza en su dimensión cultural. Por otro lado, se muestran las intenciones de conciliación entre aprovechamiento y conservación dentro de las políticas ambientales mexicanas.

Desde una perspectiva crítica a los programas conservacionistas, en general la noción de áreas protegidas, son vistas como "no lugares”. Para Sant'Ana Diegues (2000: 3):

...el parque nacional acaba por representar un hipotético mundo primitivo, intocado, a pesar de que gran parte de él haya sido manipulado por poblaciones tradicionales durante generaciones, creando paisajes de bosques mixtos, ya transformados, y otras, que raramente sufren intervenciones por parte de esas mismas poblaciones tradicionales. Esos espacios boscosos, por otro lado, pueden formar un solo paisaje. Una política conservacionista equivocada que transforma esos lugares en no-lugares, con la expulsión de las poblaciones tradicionales puede estar abriendo espacio para 


\section{http://revistainvestigacionacademicasinfrontera.com}

ISSN: 2007-8870

que esos no-lugares se conviertan en el dominio de la investigación de las multinacionales o de convenios entre entidades de investigación nacional e internacional, y al final, "privatizados" como manda el manual neoliberal.

Tal vez sea por eso que las grandes entidades conservacionistas asocien de forma tan íntima la conservación de la biodivesidad y las áreas protegidas vacías de sus habitantes tradicionales y de su cultura.

En la idea de conservación de la naturaleza mediante la creación de áreas protegidas con cualquier variante adjetiva: natural, ecológicas, etcétera, subyace el pensamiento occidental, que colisiona con la de muchos pueblos indígenas que rechazan el concepto de áreas silvestres (Zúñiga, 2006). Una de las ideas centrales modernas es la separación naturaleza sociedad, que está en la base racional de la propuesta conservacionista sobre parques nacionales en Estados Unidos durante el siglo XIX. Esta idea representa el centro de la divergencia cultural con las sociedades indígenas, donde existe una interconexión entre el mundo natural o el mundo sobrenatural y la organización social. En este sentido, no existe una clasificación dualista, una línea divisoria rígida entre lo "natural" y lo "social”, al contrario, existe un continuum entre ellas (Sant'Ana Diegues, 2000).

En sus estudios antropológicos en el Amazonas, Descola (1998) afirma que las cosmologías indígenas no hacen distinciones ontológicas entre humanos, de un lado, y un gran número de animales y humanos de otro. El autor enfatiza la idea de interconexión entre esas especies por un vasto continuum, gobernado por el principio de la sociabilidad, donde la identidad humana, vivos o muertos, de las plantas, de los animales y de los espíritus es completamente relacional.

A partir de los diálogos entre antropología y las ciencias naturales, se abren fértiles líneas de investigación que abordan el reto de la incorporación del componente social en la construcción de conocimiento, por ejemplo, generar inventarios realmente participativos, con plena anuencia y cooperación de las poblaciones, que tradicionalmente maneja la biodiversidad. Sin embargo, llevar a cabo esta empresa resulta muy compleja precisamente porque implica las amplias dimensiones de la vida social, económica, política y cultural. 


\section{http://revistainvestigacionacademicasinfrontera.com}

ISSN: 2007-8870

Las UMAs en México lograron la conjunción entre manejo y conservación incorporando a los actores sociales, frecuentemente los menos privilegiados. Es una estrategia territorial, que agrega valor a la vida silvestre bajo un esquema de apropiación social de la biodiversidad. Las UMAs nacieron formalmente el 5 de junio de 2000 en Hampolol, Campeche, dentro de la Estrategia Nacional para la Vida Silvestre (Robles de Benito, 2009: 12-13). Sin embargo, la aplicación de las políticas públicas siempre encuentra en el ámbito institucional-administrativo serias dificultades, a pesar de que documentalmente expresen la respuesta, que resolverá los problemas. Dadas las condiciones presupuestales y burocráticas de las instituciones encargadas de las políticas ambientales, su ejecución recae en instrumentos administrativos incapaces de asegurar la plena incorporación de las comunidades indígenas en el aprovechamiento y conservación de los recursos de su territorios. En este sentido, el énfasis de la política ambiental sigue pesando más sobre la conservación.

\section{$\underline{\text { Ixtepeji en la selvicultura comunitaria }}$}

La conservación de los recursos naturales es un eje nodal en la política pública forestal de México, sin embargo, para que se logren resultados reales, requieren de la participación decidida de las comunidades, dueñas del territorio que los contiene. Existen ejemplos exitosos de que el trabajo comunitario, la participación social y la política pública forestal pueden impulsar el desarrollo de los dueños y poseedores de los bosques, contribuyendo a mejorar su calidad de vida y a preservar los ecosistemas forestales (CONAFOR, 2007). Ello implicaría pasos firmes hacia la sustentabilidad, que guía la política ambiental mexicana.

El manejo forestal comunitario es realizado por las diferentes poblaciones, ejidos y grupos indígenas mexicanos, dueños de sus bosques, ya sea con fines de autoconsumo, o bien, constituidos en Empresas Forestales Comunitarias (EFC) con objetivos de producción industrial y con diferentes niveles de integración. Sus miembros poseen y comparten colectivamente los terrenos boscosos y sus beneficios, desarrollando en ellos diferentes actividades: agricultura, ganadería, silvicultura, recolección, entre otras, participando, además, en los procesos de toma de decisiones y definición de políticas 


\section{http://revistainvestigacionacademicasinfrontera.com}

relacionadas (Merino et al., 1997). Para Marshall et al. (2006), las reglamentaciones comunitarias a veces son más efectivas que la legislación nacional en cuanto al logro del uso sostenible de los RFNM.

Uno de estos ejemplos de éxito de la selvicultura comunitaria es Santa Catarina Ixtepeji, Ixtlán, Oaxaca, donde el cuidado del bosque y el trabajo en las empresas comunitarias son producto de las luchas que en el Sierra Juárez se desarrollaron en los años finales de la década de los años 70, y lograron que en 1983 no se renovaran las concesiones forestales a la empresa Papelera Tuxtepex, así como de la organización comunitaria y el apoyo de universidades y programas públicos enfocados al fortalecimiento de las capacidades técnicas de los pueblos para hacerse cargo de la conservación y aprovechamiento de sus bosques. Tras treinta años de manejo comunitario de los bosques, Santa Catarina Ixtepeji forma parte de las comunidades que desarrollan exitosamente la selvicultura comunitaria.

\section{Materiales y Métodos}

El lugar del estudio etbotánico fue Ixtepeji, que se localiza en la Sierra Norte de Oaxaca, México. Está formada por 21,691.90 ha. Se divide geográficamente en la cabecera municipal Santa Catarina Ixtepeji y cuatro agencias El Punto, Tierra Colorada, Yuvila y San Pedro Nexicho. El número total de hogares en Santa Catarina Ixtepeji es de 596. La población total del municipio es de 2,633 personas, de las cuales 1,267 son hombres y 1,366 son mujeres. Las principales actividades económicas corresponden al sector agropecuario-forestal, la agricultura y ganadería son de autoconsumo, destaca la producción y el control forestal, dentro de ésta los productos forestales no maderables como maguey de encino, maguey de pino, heno gris, heno blanco, entre otros, (INEGI, 2015).

Ixtepeji es uno de los municipios que más venden bromelias epífitas en los mercados de la ciudad de Oaxaca; cuenta con el permiso de aprovechamiento de 18 especies forestales no maderables de bromelias epífitas, y dos Unidades de manejo para la conservación de la vida silvestre (UMA), para aprovechar las dos especies registradas en la Norma Oficial Mexicana (NOM-059-SEMARNAT-2010), presentes en su territorio. Dichos documentos 


\section{http://revistainvestigacionacademicasinfrontera.com}

ISSN: 2007-8870

fueron emitidos por la Secretaría de Medio Ambiente y Recursos Naturales (SEMARNAT); la UMA "Catopsis" (Ex - 00011 -Oax), permite la colecta de las especies Tillandsia carlos-hankii y Catopsis berteroniana, y la UMA “Bromelias" (INT-105-OAX), autoriza la venta de plantas.

\section{$\underline{\text { El estudio }}$}

El estudio etnobotánico sobre las bromelias en Santa Catarina Ixtepeji indagó los saberes del uso de las plantas, la percepción que tienen de ellas, su importancia y relación con el bosque, así como el compromiso para su cuidado, en las generaciones infantiles y juveniles, ya que serán éstas quienes conserven y cuiden, o no, el recurso en años próximos.

Se realizaron doce talleres de dibujo y cuento entre julio y agosto de 2015 con los niños y jóvenes de las primarias y telesecundarias de las tres agencias y de la cabecera municipal: Santa Catarina Ixtepeji, y en tres agencias: El Punto, Tierra Colorada y Yuvila. De acuerdo al número de alumnos en cada escuela, se agruparon por grados. En total, fueron ocho talleres en primarias y cuatro en telesecundarias.

Todos los talleres se iniciaron con una motivación sobre historias de su región para sensibilizar a los participantes para narrar historias que ellos conocieran sobre las bromelias. En primaria, se organizaron equipos de siete participantes y se les pidió que elaboraran un cuento sobre lo que saben de las bromelias, sus nombres comunes, usos, importancia y lo que piensan de ellas. En las telesecundarias, también se formaron equipos de cinco adolescentes y se les solicitó que escribieran una carta a un amigo lejano describiendo los bosques de su comunidad y, en especial, las bromelias para motivarlo a visitar su pueblo. Se les proporcionó materiales para que realizaran su cuento o carta, y se les motivó a incorporar dibujos en éstos. Mientras los niños trabajaban en la elaboración de sus cuentos o cartas, se realizaron conversaciones informales para esclarecer el sentido de lo que estaban dibujando, de las historias que narraban, y si eran hijos de colectores de bromelias.

Se realizó una matriz de análisis de contenido a partir de los cuentos y cartas realizados por los niños y adolescentes con el propósito de responder a la pregunta: ¿Cuál es 


\section{http://revistainvestigacionacademicasinfrontera.com}

ISSN: 2007-8870

el conocimiento y percepción de los jóvenes y niños de las bromelias y, por ende, su compromiso para conservarlo?

\section{Resultados y discusión}

Con base en el análisis de los cuentos y cartas elaborados por los niños y adolescentes, así como las conversaciones con ellos durante el desarrollo de los talleres, se encontró que la mayoría conoce las bromelias con el nombre de magueyes, que crecen en las ramas de los árboles, que algunos tienen flores bonitas, que otros no, que algunos tienen hojas gruesas y otros delgadas.

Sobre los usos que aparecen en los cuentos y cartas, los niños y adolescentes manifestaron que principalmente los magueyes sirven como alimento para los animales como los toros; que se cortan para venderlos en Oaxaca, y que sirven de vivienda para otros animales como las ranas, mariposas y las arañas.

Sobre la importancia de las bromelias y su cuidado, los niños y adolescentes expresaron que no se deben cortar porque son parte del bosque, de la naturaleza; que "por cortarlas se están acabando"; que están en peligro de que desaparezcan; por ello, las autoridades, el Comisariado y la SEMARNAT, castigan a los que las cortan; que está prohibido cortar árboles y ellos piensan que esto está bien porque así se conserva el bosque; que se deben evitar incendios y castigar a los que los ocasionan. A partir de estas percepciones de los niños y adolescentes, se reflexiona principalmente en los peligros de asociar la conservación con la prohibición.

\section{$\underline{\text { Peligros de pensar la conservación como prohibición }}$}

Uno de los resultados más interesantes que surgió en los talleres es la respuesta de varios niños ante la pregunta de que si sus padres eran colectores; respondían: "No, eso lo prohíbe la SEMARNAT y puede castigarlo el Comisariado". "No, por sacar plantas, el Comisariado los mete a la cárcel”. Por la experiencia y contacto con varios de ellos, se sabe que sus padres efectivamente se dedican a colectar diferentes recursos según la temporada; el más común es la colecta de bromelias en diciembre, ya que forman parte de la tradición de poner 


\section{http://revistainvestigacionacademicasinfrontera.com}

ISSN: 2007-8870

nacimientos, poner el Belén. Las bromelias se usan como adorno en ellos. Los colectores encuentran en esta temporada una fuente de ingreso al venderlas en los mercados de la ciudad de Oaxaca. La recolección de bromelias representa una actividad económica complementaria para la subsistencia de los colectores.

En el estudio de Mondragón y Villa (2008), también se reportó una correlación positiva entre la edad de los recolectores y conocimiento de las plantas; es decir, a mayor edad, se cuenta con más años dedicados a la recolección y, por tanto, se conocen más especies y usos de las plantas. Esta investigación mostró la consciencia de los colectores por el cuidado de las bromelias, puesto que forman parte del bosque y consideran su deber cuidarlas, lo cual es congruente con el sentido de las luchas de las comunidades serranas por la cancelación de las concesiones forestales previas a los años 80 .

Resulta muy interesante reflexionar en las respuestas de los niños en tanto incidir en la negación de una actividad tradicional. Se advierte una transición muy importante entre generaciones en cuanto a la percepción del cuidado de las bromelias: para los colectores adultos son parte del bosque, conocen más especies, sus usos, reconocen los climas de las diferentes regiones de su territorio porque tienen más contacto con el bosque (Mondragón y Villa, 2008). En tanto que para los niños y jóvenes, las bromelias son plantas que no se deben tocar porque: "el Comisariado pone multas a quien lo hace", o porque "la SEMARNAT lo prohíbe".

Es muy relevante este dato porque hay un desplazamiento de quién es el sujeto que decide la manera del cuidado del bosque: en generaciones adultas está en ellos, en la población, en la gente de la comunidad, que conoce y cuida sus recursos, pero en las generaciones infantiles y juveniles pasa a verse como una imposición de las instituciones y entidades de autoridad, que son quienes deciden y señalan lo que puede, o no, aprovecharse del bosque en aras de privilegiar la conservación. La asociación conservación-prohibición puede ser una consecuencia del papel superior que juegan las instituciones federales sobre las locales, que finalmente tienen la prerrogativa de la ley, de reglamentaciones operativas, que rigen las actividades referentes al aprovechamiento de los recursos naturales maderables y no maderables. 


\section{http://revistainvestigacionacademicasinfrontera.com}

ISSN: 2007-8870

\section{Alejamiento del bosque}

Pensar la conservación a través de la reducción a la prohibición de las autoridades federales, operada por las locales, implica reducir el acceso a sus territorios, y por ende, al conocimiento, que generaciones adultas tienen de ellos. Limitar el contacto con el recurso es un peligro importante para la continuidad de la relación directa con los elementos del entorno natural.

La fuerza, que subyace a la defensa que las comunidades dieron por la cancelación de las concesiones forestales, está en el vínculo estrecho que los pueblos zapotecos de la Sierra han tenido con el bosque, con sus tierras, con los poblados vecinos. La defensa parte de esta relación cercana con la naturaleza, de su conocimiento de los senderos, de las plantas, animales, de las variaciones del clima en su territorio. Bosque encierra muchos elementos importantes para la reproducción de la vida social, tanto material como simbólica.

El conocimiento fino sobre cuál planta sirve para qué enfermedad, cuáles hongos son comestibles, en qué temporadas se encuentran, de dónde se puede obtener un buen abono, dónde la leña, etcétera, son importantes para las comunidades humanas porque les sirven para el sustento de la vida, para intercambiar determinados productos: representan un sentido material, necesario para vivir. Pero no solamente. También el bosque guarda otros sentidos que emana de esa relación estrecha. Por ejemplo, existen en la Sierra historias de que "al monte se le pide permiso" para cortar árboles, o de que "el viento habla", que "el monte avisa" si es día para cortar o no. El monte, el viento no son cosas, no son recursos que se pueden tomar simplemente; sino seres con los que se tiene una relación de intercambios.

De ahí que la sutil prohibición de la colecta, de que no se deben tocar porque se están acabando puede estar también incidiendo en el alejamiento del bosque y, por ende, del riquísimo conocimiento que hay sobre él. Esto resulta muy preocupante para pensar en el sentido que se va constituyendo en las generaciones infantiles y juveniles, quienes en pocos años más tendrán la responsabilidad de continuar o no el cuidado y la defensa de sus territorios. 


\section{http://revistainvestigacionacademicasinfrontera.com}

ISSN: 2007-8870

Dado que Ixtepeji es un pueblo zapoteca, con un manejo comunitario del bosque, se cree que es necesario poner atención en estas perspectivas de la conservación muy asociadas a la admisión de la prohibición externa al aprovechamiento de sus recursos. Será necesario diseñar estrategias que permitan que el cuidado de un recurso no limite el contacto y el cultivo de la relación con él para asegurar la trasmisión de los conocimientos materiales y simbólicos que constituyen su patrimonio biocultural.

\section{A manera de conclusión}

El estudio etnobotánico muestra aspectos que debe analizarse con profundidad: por un lado, se advierte el conocimiento general que tienen sobre las broemelias y que es menor que el de los adultos, reportado por Mondragón y Villa (2008), lo cual coincide con sus resultados; es decir, que a menor escolaridad de los colectores, conocen más especies y usos, precisamente porque tienen más años dedicados a la recolección. Por otro lado, resulta muy importante reflexionar en uno de los resultados del estudio: la expresión en los niños y adolescentes sobre la prohibición y sanción por parte de las autoridades comunales y las instituciones federales (SEMARNAT), ya que muestran la adopción del discurso por la conservación, sin embargo, el sujeto que decide y manda es principalmente externo: la institución de gobierno federal sobre la autoridad comunal, es decir, el Comisariado ejecuta las sanciones, pero quien impone la prohibición es la SEMARNAT.

En este sentido, se advierte un desplazamiento muy importante porque en el estudio de Mondragón y Villa (2008), la percepción del cuidado de las bromelias para los colectores adultos está en ellos, como los sujetos, dueños del bosque y comprometidos con su cuidado y conservación. En tanto, que actualmente los niños y adolescentes admiten como positivas la prohibición y las sanciones como formas para conservar el bosque y perciben a las instituciones federales como los sujetos que dirigen y ordenan este proceso. Por ello, pensamos que debe tomarse muy en cuenta este aparente cambio sutil, porque puede implicar una forma de enajenación del cuidado del bosque, al moverse el sujeto que posee el bosque y, por tanto, está comprometida con su cuidado: la comunidad; a que ésta siga poseyendo el bosque, pero deba seguir las directrices externas por parte de las instituciones federales en la 


\section{http://revistainvestigacionacademicasinfrontera.com}

ISSN: 2007-8870

ejecución de las políticas públicas para su conservación. Es decir, quien decide qué conservar y cómo hacerlo es ahora un agente externo y no ya la misma comunidad.

\section{Literatura citada}

Anglés-Hernández M. (2008). El Desarrollo Sostenible al centro de la tríada: Pobreza,

Medio Ambiente y Desarrollo. Revista de Direito Ambiental. 13(50):306-327.

CONAFOR. (2007). Experiencias de manejo forestal comunitario. Opción Gráfica Integral, México.

Descola, Philippe. (1998). Las comologías de los indios de la Amazonía. Zainak, 17:219227.

Diario Oficial de la Federación. Ley General Del Equilibrio Ecológico y la Protección al Ambiente. 28 de enero de 1988.

Diario Oficial de la Federación. Ley General de Desarrollo Forestal Sustentable. 25 de febrero de 2003

Diario Oficial de la Federación. Ley General de Vida Silvestre. 3 de julio de 2000.

Diario Oficial de la Federación. Ley Federal Sobre Metrología y Normalización. $1^{\text {o }}$ de julio de 1992

Gallina-Tessaro S, Hernández-Huerta A, Delfín-Alfonso C, González-Gallina A. (2008). Unidades para la conservación, manejo y aprovechamiento sustentable de la vida silvestre en México (UMA). Retos para su correcto funcionamiento. Investigación Ambiental 2: 143-152.

Anuario estadístico y geográfico de los Estados Unidos Mexicanos 2016. (2016). Instituto Nacional de Estadística y Geografía. México.

Marshall E, Schreckenberg K, Newton AC. (2006). Comercialización de Productos Forestales No Maderables: Factores que Influyen en el Éxito. Conclusiones del Estudio de México y Bolivia e Implicancias Políticas para los Tomadores de Decisión. Centro Mundial de Vigilancia de la Conservación del PNUMA, Cambridge, Reino Unido. 


\section{http://revistainvestigacionacademicasinfrontera.com}

ISSN: 2007-8870

Merino, L. (Coord.) et al. (1997). El manejo forestal comunitario en México y sus perspectivas

de sustentabilidad. UNAM, SEMARNAP, CMSS, México.

Mondragón, D. (2008). La comercialización navideña de bromelias epífitas en la ciudad de Oaxaca, México. Etnobiología 6: 24-28.

Mondragón, Demetria, Villa-Guzmán, Dulce María.(2008). Estudio etnobotánico de las bromelias epifitas en la comunidad de Santa Catarina Ixtepeji, Oaxaca, MéxicoPolibotánica [en linea] 2008, (octubre) : [Fecha de consulta: 4 de enero de 2016] Disponible en:<http://www.redalyc.org/articulo.oa?id=62102610> ISSN 1405-2768

Profepa. (2000). Las cactáceas, bromelias y orquídeas en peligro de extinción. PROFEPA Delegación Oaxaca, México.

Rauh, W. (1992). Are Tillandsias endangered plants? Selbyana 13: 138-139.

Rees, J. (1976). The Oaxaca Christmas plant market. Journal of the Bromeliad Society Bulletin 6: 223-232.

Robles de Benito, R. (2009). Las unidades de manejo para la conservación de vida silvestre y el Corredor Biológico Mesoamericano México. México, CONABIO Corredor Biológico Mesoamericano México, Serie Acciones / Número 2.

Sant'Ana Diegues, A. C. (2000). Etno-conservación: Conocimiento y Manejo Tradicionales - Ciencia y Biodiversidad. NUPAUB-USP, São Paulo.

Wolf, J.H.D \& C.F.J. Koning. (2001). Toward the sustainable harvesting of epiphytic bromeliads: a pilot study of the highland of Chiapas, Mexico. Biological conservation 101: 23-31

Zúñiga García-Falcés, N. (2006). Conflictos por recursos naturales y pueblos indígenas. Pensamiento Propio, No 22, julio-diciembre de 2006.

\section{Agradecimientos:}

Al Instituto Politécnico Nacional a través de la Secretaría de Investigación y Posgrado por el apoyo al proyecto de investigación: "En la búsqueda del aprovechamiento sustentable de Tillandsia carlos-hankii en Santa Catarina Ixtepeji". Clave: SIP20151017 
Año 10, Núm. 25 (Enero - junio 2017)

Revista de Investigación

Académica sin Frontera ISSN: 2007-8870

http://revistainvestigacionacademicasinfrontera.com 